\title{
THE SUCCESS STORY OF MITRA TRANSLATIONS
}

\author{
Mina Ilieva ${ }^{1}$, Mariya Kancheva ${ }^{2}$ \\ ${ }^{1}$ CEO at Mitra Translations, \\ ${ }^{2}$ Lead Linguist at Mitra Translations, \\ 1mki@mitra-bg.com \\ ${ }^{2}$ mariya_kancheva@abv.bg
}

Mitra Translations was found back in 1989 as a small local translations business in Shumen, Bulgaria. The founder, Teodora Todorova, was then a teacher in Bulgarian language and literature and Russian as well. Her strong ambition about literature and languages motivated for establishing a business to serve people in their efforts to communicate and exchange knowledge in different languages. That time was historical for Eastern European countries and started to blow the so called "wind of change". That decade resulted in a huge migration, respectively it lead to the need of translation services. That was the first wave to the successful steps to go ahead. We remember the times of the hegemony of the typewriter and the revolution that came with computer popularization. Now, we are contemporaries of the software innovations, machine translation and the AI era, and we are eager to find out about what else the future has to offer.

Technologies and their constant updates and innovative nature drastically and irreversibly transformed this small business into a leading brand on the translation market, along with just few other LSPs integrating translation software solutions. Now, we are constantly following the new developments in software updates and online platforms and we are successfully keeping up with any new trend in the field of translation, localization, transcreation, revision, post-editing, etc. Nowadays, in Bulgaria $95 \%$ of the translation agencies have never heard of or integrated any CAT and MT tools, that is why we managed to get within the TOP 3 translation brands on the national market, because we choose to go the "hard" way. I am saying hard as there are still lots of colleagues and translators who believe that the use of technology is quite complicated and not so useful, also time and cost effective.

Introducing technologies into our everyday work brought a lot of benefits but it also made us face certain challenges. One of them was that the majority of the translators we worked with were not capable to operate or were not very confident in using the new platforms, software and web-based solutions. This was a crucial moment, a milestone which we realized would in fact give us a great advantage. This was the point when we started training our own in-house translators how to use the CAT tools and new software. We knew it from the beginning that if we wanted to succeed in the industry, we had to be ready to invest in technology and in our staff knowledge and skills. The strategy paid back really well. At the end we managed to both meet the expectations of our 
international clients and build a competent bank of translation professionals. Basically, we provide training for our in-house translators in using CAT tools by introducing simulations and real time projects. In addition translators find webinars a useful and easy way to maintain their CAT and MT tools knowledge. They have also shared with us that while following innovations they felt wellqualified and competitive among their fellow professionals. Later on, when our trained in-house resource started working for us on a freelance base, it was easier than ever to engage competent vendors for our increasing number of projects. Today, we rely on 10 qualified in-house translators and $200+$ freelance translators which add up for covering the total demand regarding all EU and Asian languages. Furthermore, we did presentations at universities in Bulgaria to make students familiar with the idea of "contemporary profession of translator" focusing on technologies and how to make use of them. It felt almost like a mission. This encouraged us to eventually devote further efforts into a training programme. In 2016 we participated in the EU Operative Programme for Human Resource Development. As part of that project we trained quite a lot of students from a few universities in Bulgaria and received positive feedback for our endeavors to bridge the gap between university and business. Students showed immediate progress and passion for new technologies. We would say that along with universities, today, we are more than certain that young professional who choose the translator's career need our support as well in bringing their work to the next level.

On the one hand we experience the problem with the shortage of qualified translators ready to work with translation technologies and on the other hand there is the problem with the shortage of qualified trainers as part of the reality in Bulgaria. The only authorized reseller of SDL Trados offers a "getting started" version of training after paying for a license. Evidently there are not enough trainers, either in practice or theory, to prepare qualified translators to use translation tools and software; and SDL Trados surely is not the only CAT tool the industry happens to require. Many believe that getting the hack of SDL Studio will be enough to use the other tools and platforms. This could be somewhat true due to the fact that the operation logic of two or more types of translation software can be similar. However we have heard some translators say that apart from Trados they were not able to "understand" or be confident with using other CAT tools. Yet another problem is that in general freelancers are not eager to pay license fees, they would not invest their time in additional training, and quite naturally they are reluctant to pay for more than one software.

The translation industry in Bulgaria lacks trained professionals in the field of DTP as well. We faced this problem in 2012 while working for a large translation project regarding huge power plant instructions for use. The translated files were ready, however the only two DTP service providers, who just to mention worked at advertising companies, offered a relatively high price for the pace of 4 pages per day. Deadline was pressuring us and we were forced to seek DTP providers based in China who luckily managed to do the task cheaper, for $10 \%$ of the price quoted in Bulgaria, and more 
efficiently, processing 4 pages per hour. We believe that training in the translation industry should be thought as intended industry strategy rather than existing as individual instances among companies.

Our experience at a global level confirmed that professionalism speaks a universal language. Deadlines, budget and quality are equally important to stand out from business competitors. Once again technology helps fulfill these pre-conditions and presents greater opportunities for expanding our client network. Usually each project requires the use of different tools as well as certain clients prefer project delivery in a given platform, software or format. Diversification of CAT and MT tools and web-based solutions for translation and QA is yet another demand for the business. The specialized software and programme mainly used within the company are SDL Trados Studio 2019, SDL Trados GroupShare 2015, MemoQ (cloud based server option), xtm, Translation Workspace, Wordfast, Memsource, etc. As seen from the pie chart below, SDL Trados Studio has the largest share among the four most used softwares at our company. The tendency has remained unchanged during the years with prevalence of SDL Studio, followed by MemoQ, and Wordfast and Translation Workspace with an almost equal share.

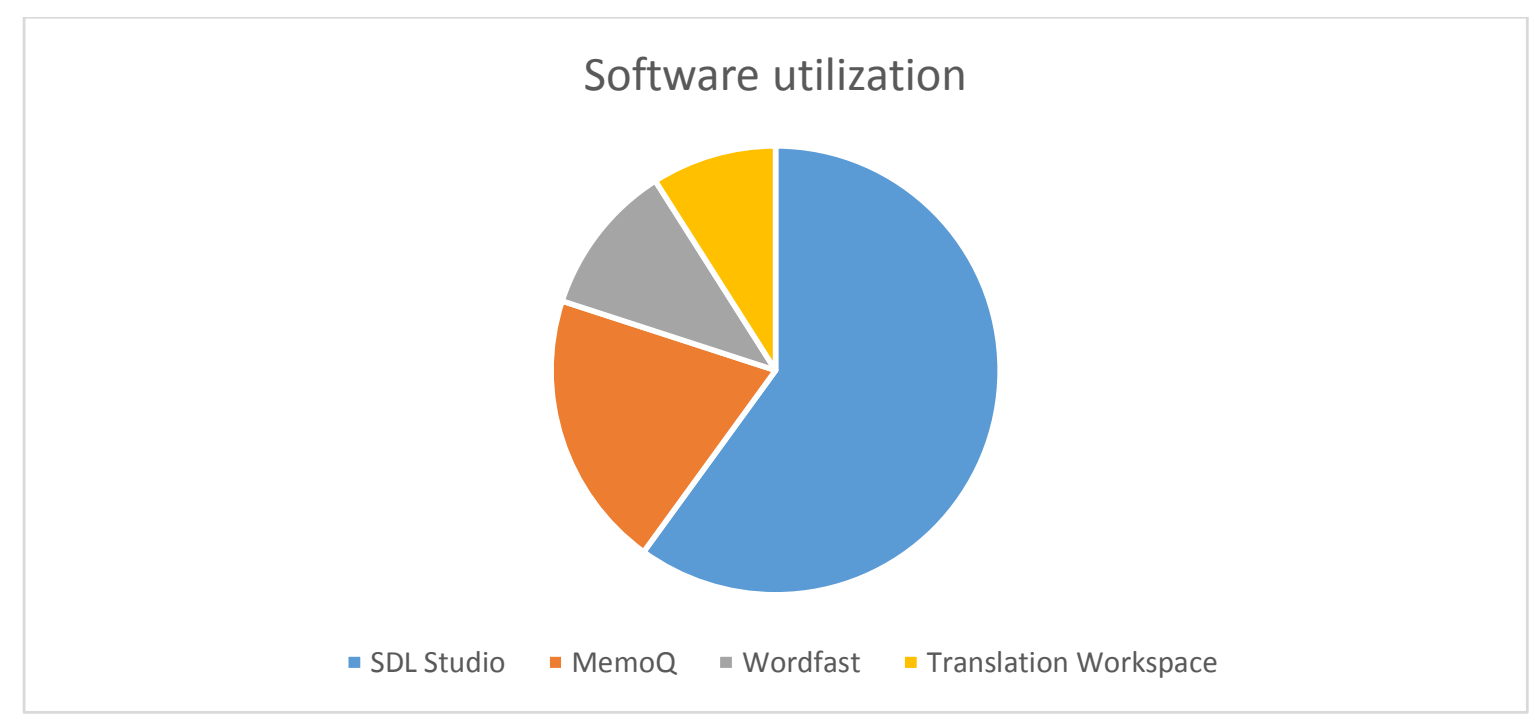

Fig. 1. Types of software utilization at Mitra Translations

Our analysis leads to the conclusion that this spread is due primarily to clients' preferences and requirements. Popularity of SDL Trados Studio among vendors might also come from the good promotion of the software in Bulgaria and the user-friendly interface. Updates are readily available almost every year and new features are added to accelerate and ease translators' work. However, since time is of great significance we must be ready to operate with any software/ online tool in advance. 
Project management is another aspect of our workflow which benefits from technology implementation. File analysis and project management were also assisted by web-based TMS and CAT tools we use. The entire project workflow became more clear and structured, while time was reduced to a minimum. Figure 2 shows a breakdown of one of our projects which includes 101 files. As evident from the figure, $48.2 \%$ from the total wordcount $(86,453)$ are repetitions, and $13.5 \%$ represent cross-file repetitions. Back in the days with no CAT tools utilization it would have taken much more time to complete this job, and consistency would probably suffer. However, again, project managers get trained internally at our company.

\begin{tabular}{|c|c|c|c|c|c|c|}
\hline 22 Total & Type & Seaments | & Words $C$ & Characters & Percent & \begin{tabular}{|l|l} 
Recognized Tokens Tags \\
\end{tabular} \\
\hline 23 Files:101 & PerfectMatch & 0 & 0 & 0 & $0.00 \%$ & 0 \\
\hline 24 Chars/Word:4.61 & Context Match & 0 & 0 & 0 & $0.00 \%$ & 0 \\
\hline 25 & Repetitions & 7967 & 41683 & 179009 & 9. $48.21 \%$ & 8379 \\
\hline 26 & Cross-file Repetitions & 4906 & 11700 & 48249 & $913.53 \%$ & 2902 \\
\hline 27 & $100 \%$ & 0 & 0 & 0 & $0.00 \%$ & 0 \\
\hline 28 & $95 \% \cdot .99 \%$ & 0 & 0 & 0 & $0.00 \%$ & 0 \\
\hline 29 & $85 \%-94 \%$ & 0 & 0 & 0 & $0,00 \%$ & 0 \\
\hline 30 & $75 \% \cdot 84 \%$ & 0 & 0 & 0 & $0.00 \%$ & 0 \\
\hline 31 & $50 \% \cdot 74 \%$ & 0 & 0 & 0 & $0.00 \%$ & 0 \\
\hline 32 & New & 9110 & 33070 & 171393 & $38.25 \%$ & 5098 \\
\hline 33 & Total & 21983 & 86453 & 398651 & $100 \%$ & 16379 \\
\hline
\end{tabular}

Fig. 2. File analysis, SDL Trados Studio 2017

Eventually our business analyses show that after integrating CAT tools in our translation and project management process our productivity increased to more than $50 \%$. Mitra's experiences show that CAT tools cut the time devoted to the whole output and made translation up to $50 \%$ faster and therefore more time-efficient, moved translators' work from typing to actual translating which accelerated the translation process. The cost for the end client decreased because now we can use file analyses and automatically and precisely count repetitions, fuzzy matches and no match entries which are paid at different rates. These benefits along with the fact that international LSPs seek company vendors who are able to meet their requirements in terms of translation technology utilization, they all logically lead to better business results with Mitra increasing with $10 \%$ sales growth every year for the past few years.

As far as quality is concerned we try to incorporate any relevant QA check feature of the translation tools at our company. It saves hours of review time and boosts productivity. However, we could not help but notice that sometimes more than one QA tool should be used to eliminate certain errors. If 
for an identical bilingual translation file an LTB report and an SDL QA check are run, the user may find stunning discrepancies in the number and nature of reported errors and mistakes. It often helps to run for example two QA tools, however this is extremely time consuming for large texts and means extra work which goes beyond client's expectations, requirements and expenses.

Training translators and project managers, the variety of programmes which we had to introduce into our work due to business demand, and the need of applying multiple QA tools in order to get a "clean" QA report have so far been some of the challenging points related to technology implementation in our success story.

Ultimately, we are positive that proper implementation of technology (with focus on quality, cost and time) and hard work are the stepping stones in the way to become a trusted language service provider. 